ORIGINAL ARTICLE

\title{
Comparison of oxygen consumption of football players in different positions of the game
}

RAMAZAN CEYLAN ${ }^{1}$

${ }^{1}$ Bayburt University, Sport Sciences Faculty, BAYBURT, TURKEY

Correspondence to Dr Ramazan Ceylan, Email. rceylan@bayburt.edu.tr, Cell: 5055899335, orcid:0000-0003-0123-0783

\begin{abstract}
Background: Football players have different structural and physiological characteristics due to the variety of tasks that lie in this field. The main purpose of this research was to compare oxygen consumption of football players in different positions of the game.

Materials and methods: The research method was causal or post-occurrence. So nine halfbacks, seven attackers, three goalkeepers and six defenders were chosen to participate in this research.

The Chinese bicycle was used to measure Vo2max. The heart rate was determined by the electronic heart rate. Nolan Margaria-Kalamen test machine was used to measure the anaerobic power of players. Analysis of variance and $\mathrm{F}$ test were used to compare the mean of differences. All steps were performed using SPSS 21 at the level of 0.05 .

Results: There is a significant difference between Vo2max of goalkeepers and halfbacks; between goalkeepers and attacking players; between defenders and attackers. There was a significant difference between the anaerobic capacity of halfbacks and goalkeepers; between attackers and goalkeepers.

Conclusion: The position and the duration of activity has a great effect on $\mathrm{Vo}_{2 m a}{ }^{x}$ and anaerobic of players. So to increase both characteristics of the players depends on the position of the game.

Key words: oxygen consumption, anaerobic consumption, football, positions of the game.
\end{abstract}

\section{INTRODUCTION}

There is no doubt that football is one of the most popular sports in the world and can be that to the scene of interesting and exciting the millions of people have charmed and fascinated by his departure and they have to watch it draw the track.1,13

The increasing development of sports science, which is achieved through the valuable research and experiences of researchers, has led to the specialization of various branches and disciplines of sports and the evolution of training programs for different teams. Although in our country, positive steps have been taken in the development of football, but it is not enough, and some obstacles and shortcomings, especially in the fields of education and research, have caused our country's football from most European and African countries and even some Is also behind Asian countries. ${ }^{2}$

One of the prominent aspects of progress and success in any sport is recognizing the effective factors in the championship and also the coaches' awareness of the physiological features and functional capacities effective in the victory and success of their athletes. For this reason, it is obvious that the lack of knowledge of the factors affecting sports success and the lack of awareness of coaches about the abilities and capabilities of athletes will lead to lack of progress and waste energy and time and will lead to exorbitant costs. ${ }^{3,12}$

Football players have different structural and physiological characteristics due to the variety of tasks that lie in this field. Coaches should know the characteristics and characteristics of each of their players and according to the role and duty of each person, develop a good training program and preparation for them. Physical education experts believe that all exercise programs should be designed and implemented in a way that develops specific physiological capacities that require specific skills or activities. ${ }^{4}$

According to the principle of movement training on a special energy device, more pressure is applied. The potential for further performance improvement in sports activities that include that energy device can be expected. Believing in this principle, it is important that all coaches should try to adjust the training program of the champions based on and sometimes on the anatomical and physiological characteristics of their bodies for sports success, and give the athletes morning exercises in order to gain physical fitness and training. Their structural and physiological characteristics are consistent. ${ }^{5}$

Due to the number of roles and tasks in the game of football and because the players of this field need special physical abilities to perform the assigned tasks, it is necessary to conduct a clearer view of the current situation of our country's players by conducting laboratory and experimental research. Based on the results of such researches and comparing them with similar researches in other countries, we should be aware of the current trend of training methods and selection of club players, and when we move with successful teams in the world. ${ }^{6}$ The measurement of capabilities and effective factors in the novel category of football teams, followed by the knowledge and awareness of the status of players in the club, national and international categories in different countries, has been done many times, while the implementation of such projects on Turkish footballers is very small. ${ }^{7}$

By clarifying the physiological indicators of the players and comparing them with each other and with external criteria, the necessary guidelines can be provided to the coaches of this field so that they can use these guidelines to develop more involved programs to equip their teams. On the other hand, players can be aware of their characteristics and adapt them to the task they are responsible for in the game, receive 
the necessary feedback to improve and develop their performance capacity as much as possible and gain the necessary motivation to continue training.

\section{MATERIAL \& METHODS}

In this research, the researcher seeks to explore the relationship between factors and conditions that have occurred before and has no involvement in the occurrence or manipulation of variables and is involved in measuring several variables (dependent on maximum oxygen consumption and anaerobic capacity) and by relating them to several independent variables (football game posts), to achieve the accuracy and robustness of research hypotheses. Therefore, the research method is causal or post-occurrence.

Statistical Society: The statistical population includes players who were members of one of the first division clubs in Turkey in 2020. And participated in the national competitions of the Turkish League

A: halfbacks: This group includes 9 midfielders who played as halfbacks in their team.

B: Attackers: This group includes 7 players who played in the offensive line in their club team.

C: Goalkeepers: This group includes 3 goalkeepers who stand in the goal.

D: Defenders: This group includes 6 players who played in the back position.

\section{Tools and instruments used in research}

1- $\quad$ Monark bike Ergometer model 894E was used to measure the maximum oxygen consumption. The heart rate of the subjects during the activity was determined by the electronic heart rate recording device. ${ }^{8}$

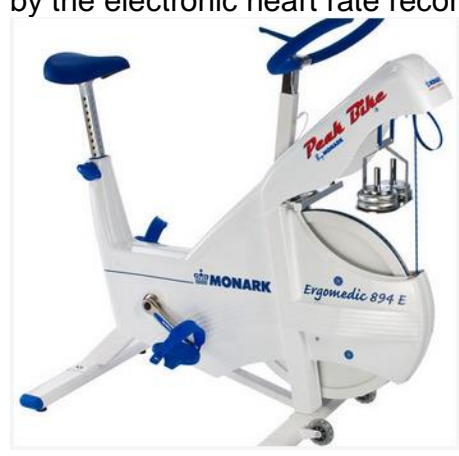

Fig1. Monark bike Ergometer

2- Margaria-Kalamen climb stair test was used to measure the anaerobic power of players. ${ }^{8}$



Fig 2. Margaria-Kalamen climb stair
This device includes a metal staircase in the row of stairs. On the third and ninth steps there is a drive plate that connects to an electronic timer with an accuracy of 0.01 seconds.

3- Seiko calculator model 3800 * Fx K was used for statistical calculations.

\section{A personal computer}

Steps of conducting research: In order to gather the required information and perform the predicted tests, the selected players were invited to attend the Sports Physiology Laboratory. Then, explanations were given on how to perform the tests and its various stages, and the points that should be observed by the players. After this stage, a questionnaire was given to the players. This questionnaire contained questions that were necessary to determine the desired indicators and extract the results. After filling out the questionnaire, the desired characteristics were measured.

Protocol of the work: Participants cycled for 5 minutes. The pedaling speed was $60 \mathrm{rpm}$ and the workload was $900 \mathrm{~kg}$ per minute. At the end of 5 minutes the heart rate was recorded. Oxygen consumption was calculated based on Fox Matthews formula. ${ }^{8}$

$\mathrm{HR}_{\text {sub }}=0.0193-6.3=? \mathrm{Vo}_{2 \max }$

To measure the anaerobic power of each participant, he climbed three steps and power efficiency was calculated based on the following formula ${ }^{8}$

$\mathrm{P}=\mathrm{W}^{\star} \mathrm{D} / \mathrm{T}$

Data analysis: Analysis of variance and $F$ test were used to compare the mean of differences. All steps were performed using SPSS 21 at the level of 0.05

\section{RESULTS}

Table 1. Mean and physiological standard deviation of players

\begin{tabular}{|l|l|l|}
\hline Players & $\mathrm{VO}_{2 \max }$. Consumption & Anaerobic Power \\
\hline Goal keepers & $46.24 \pm 3.12$ & $147.54 \pm 12.47$ \\
\hline Defenders & $48.19 \pm 16.2$ & $137.87 \pm 19.11$ \\
\hline Halfbacks & $52.65 \pm 12.11$ & $121.45 \pm 21.34$ \\
\hline Attackers & $56.76 \pm 3.76$ & $119.43 \pm 12.12$ \\
\hline
\end{tabular}

Table 2. Comparison of maximum oxygen consumption of players

\begin{tabular}{|l|l|l|}
\hline Players & $\mathrm{VO}_{2 \max }$ & $\mathrm{P}$ \\
\hline goalkeepers & 41.43 & 0.035 \\
\hline defenders & 48.11 & \\
\hline Halfbacks & 45.95 & 0.001 \\
\hline Goalkeepers & 50.11 & \\
\hline Attackers & 45.18 & 0.001 \\
\hline Goalkeepers & 55.01 & \\
\hline Halfbacks & 44.34 & 0.45 \\
\hline Defenders & 47.12 & \\
\hline Defenders & 42.87 & 0.001 \\
\hline Attackers & 55.12 & \\
\hline Halfbacks & 45.76 & 0.34 \\
\hline Attackers & 47.98 & \\
\hline
\end{tabular}

Table 3. Comparison of anaerobic consumption of players

\begin{tabular}{|l|l|l|}
\hline Players & Anaerobic & $\mathrm{P}$ \\
\hline defenders & 152.11 & 0.045 \\
\hline goalkeepers & 149.87 & \\
\hline Halfbacks & 157.19 & 0.001 \\
\hline goalkeepers & 132.18 & \\
\hline attackers & 142.86 & 0.001 \\
\hline goalkeepers & 162.19 & \\
\hline Halfbacks & 164.98 & 0.65 \\
\hline defenders & 159.80 & \\
\hline attackers & 159.11 & 0.057 \\
\hline defenders & 158.85 & \\
\hline attackers & 138.99 & 0.062 \\
\hline Halfbacks & 141.56 & \\
\hline
\end{tabular}


Among players, strikers have the most oxygen consumption below maximum and goalkeepers have the lowest oxygen consumption.

There is a significant difference between $\mathrm{Vo}_{2 m a x}$ of goalkeepers and halfbacks; between goalkeepers and attacking players; between defenders and attackers.

There was a significant difference between the anaerobic capacity of halfbacks and goalkeepers; between attackers and goalkeepers.

Table 4. ANOVA test for $\mathrm{V}_{2 m a x}$ participants

\begin{tabular}{|l|l|l|l|l|l|}
\hline $\begin{array}{l}\text { Source of } \\
\text { changes }\end{array}$ & $\mathrm{df}$ & $\mathrm{SS}$ & $\mathrm{SM}$ & $\mathrm{F}$ rate & $\mathrm{F}$ probable \\
\hline $\begin{array}{l}\text { Between } \\
\text { groups }\end{array}$ & 4 & 295.65 & 87.65 & 3.97 & 0.02 \\
\hline Within groups & 21 & 413.76 & 17.54 & & \\
\hline Total & 25 & 709.41 & & & \\
\hline
\end{tabular}

The results of the table show that the players are significantly different in terms of $\mathrm{Vo}_{2 m a x}$ based on the game post

Table 5. ANOVA test for Anaerobic power of participants

\begin{tabular}{|l|l|l|l|l|l|}
\hline $\begin{array}{l}\text { Source of } \\
\text { changes }\end{array}$ & df & SS & SM & $\begin{array}{l}\mathrm{F} \\
\text { rate }\end{array}$ & $\begin{array}{l}\mathrm{F} \\
\text { probable }\end{array}$ \\
\hline $\begin{array}{l}\text { Between } \\
\text { groups }\end{array}$ & 4 & 2567.76 & 1254.75 & 2.98 & 0.04 \\
\hline Within groups & 21 & 7654.67 & 298.54 & & \\
\hline Total & 25 & 10222.43 & & & \\
\hline
\end{tabular}

The results of the table show that the players are significantly different in terms of anaerobic power based on the game post.

\section{DISCUSSION}

The results show that there is no significant difference between the maximum oxygen consumption of goalkeepers and defenders. The results show that these two groups of players have the lowest values and their average aerobic capacity is calculated lower than the average total oxygen consumption of the subjects. Regarding the oxygen consumption of the goalkeepers, I must say that these players usually in the games and competitions run shorter distances than others. Therefore, it seems that they need less aerobic power than the players of other positions. On the other hand, we should note that by changing the rules of the football game, which does not allow goalkeepers to receive the balls that are sent to them by their own players with their feet, they have to force the goalkeepers to perform their duties. He has teamed up to get out of the goal to repel the ball, control the ball and pass to other players. In addition, in a number of matches, goalkeepers have been removed from the penalty area within minutes of the game, especially in the second half, and added to the players on the pitch. For these reasons, it is observed that in the team training program, goalkeepers, like other players, participate in general training, which often increases aerobic capacity. The results show that there is a significant difference between the maximum oxygen consumption of goalkeepers and midfielders. We have already mentioned that in the new football systems, goalkeepers often participate in aerobic exercises like other players. Therefore, the approach of the goalkeepers' aerobic power to other players is not unexpected. However, there is a significant difference between the oxygen consumption of goalkeepers and midfielders. Although goalkeepers try to increase their aerobic capacity, but because these players travel less distance than other players during the game and play, this has prevented them from developing or maintaining their aerobic capacity alongside other players. Most similar studies have reported similar results to this hypothesis. Burroughs and his colleagues have reported similar results for English first and second division players. In their research, goalkeepers scored the lowest vo2max, which was significantly lower than the vo2max of midfielders. The results show that there is a significant difference between the maximum oxygen consumption of goalkeepers and attackers. The attackers with the highest average oxygen consumption have a significant difference in this respect with the goalkeepers. In most studies, goalkeepers have the lowest aerobic capacity compared to other players, which is consistent with our results. But because the distance they run during the tournament is much shorter than the distance traveled by the other players, it seems natural that they have the least maximum oxygen consumption (25 and 45). This study shows a significant difference between Vo2max goalkeepers and attacking players. ${ }^{9}$

The results show that there is no significant difference between the maximum oxygen consumption of defenders and midfielders. According to the figures, the midfielders have higher oxygen consumption than the defenders. The average oxygen consumption of these players is higher than the average of the total and the average oxygen consumption of the defenders is lower than the average of the total participants in the study. Despite this difference, the aerobic capacity of these two groups is not significant. The midfielders constantly need good physical condition, especially general endurance, to perform their special tasks, the most important of which is to connect the attackers and defenders and force them to move across the width and width of the pitch. People take part in both attack and defense, they also need the necessary factors to perform the duties of these two positions. New football systems, on the other hand, require defenders to perform offensive duties in addition to defensive duties. Performing these new tasks has forced the defenders to move forward in the event of an attack and play along and across the field. ${ }^{10}$

The results show that there is a significant difference between the maximum oxygen consumption of defenders and attackers. In modern football systems, coaches place a lot of emphasis on defending the goal. According to this principle, team members are forced to constantly change their position from attack to defense and from defense to attack. In these methods, moving forward to score and then moving backwards is necessary and important to participate in defense. The reason for an attacker to go back and support defenders is to acquire the necessary aerobic ability and it seems natural that these players, like other players on a team, improve and develop their general endurance and aerobic capacity to handle roles. And the complex tasks that modern football demands today. On the other hand, the low aerobic capacity of defenders from attackers and midfielders is not justifiable and needs to be discussed separately. As we said, defenders, in addition to performing defensive duties, have to move forward in moments of the game and in times of attack. In the new systems, especially System 25_3, which has been used by most national and club teams for several years, the 
tasks of defenders, especially full-backs, have become more difficult and complicated, requiring them to move across the field and participate in the attack. Therefore, it seems logical to expect higher aerobic capacity from defenders. This is done in advanced training programs, and all players participate in general endurance training equally. ${ }^{11}$

The results show that there is no significant difference between the maximum oxygen consumption of attackers and midfielders. According to the figures obtained from the subjects, we see a slight difference between the aerobic capacity of the attackers and the midfielders. Midfielders need good physical condition, especially general and local endurance, to perform their specific tasks, the most important of which is to communicate between attackers and defenders. In addition, because these players help both attacking and defending defenders, they must have the necessary factors for the position of defense and attack. The results show that there is no significant difference between anaerobic power of goalkeepers and defenders. Goalkeepers need a high explosive power to play an effective role in the game of football. For this reason, they spend most of their training time on explosive and anaerobic exercises. Exercises such as jumping, jumping, diving and punching the ball boost their body's immediate energy resources, which is why the high anaerobic power of goalkeepers is something that can be expected from most foreign research. Defenders also constantly develop their anaerobic capacity to perform defensive tasks that often force them to jump and jump to repel balloons, as well as to fully cover their defensive area and prevent attackers from infiltrating empty spaces behind defensive lines. And it is natural that they have good anaerobic power. ${ }^{12}$

The results show that there is a significant difference between the anaerobic power of goalkeepers and midfielders. High anaerobic capacity of goalkeepers is required to play an effective role in the game of football, and special training of these players will develop this ability and capability in them. We have already acknowledged that midfielders, in addition to performing their specific duties, participate in both attack and defense, so they must develop their abilities and capabilities in parallel with other players in order to perform their duties well.

The results show that there is a significant difference between the anaerobic capacity of goalkeepers and attackers. Attacking players need excellent physical abilities and physical condition to perform well in their duties in football. As mentioned earlier, the ability to perform speed and explosive actions is one of the most essential factors for attackers. Attacked on football players in different countries, the attackers had a high GPA in tests of speed and anaerobic power. In the vertical jump test, the Greek professional players did not show a significant difference with each other. The English football players also had a similar anaerobic capacity. Among these players, goalkeepers have the highest scores and attackers the lowest, and there is a significant difference between the anaerobic capacity of goalkeepers and attackers. The attackers reportedly disagreed with the results of the study, which could be due to differences. Attackers spend most of their training time doing an aerobic training at speeds of 50-30 meters. Therefore, the nature of their training is different from that of goalkeepers. Performed by goalkeepers, it develops better. The type of tests that measure anaerobic power is also different, and this in itself can lead to variable results. ${ }^{6-8}$

The results show that there is no significant difference between the anaerobic power of defenders and midfielders. Looking at the results, we find that the anaerobic capacity of the midfielders is lower and the anaerobic capacity of the defenders is higher than the average of the total anaerobic capacity of the players participating in the study. In this regard, considering the role and duty of the players in the new tactics that eliminate the distinctions of the task and require all players to participate in attack and defense, and the result obtained is acceptable. But midfielders are expected to work harder to achieve higher anaerobic power and peak fitness. Most foreign studies have reported similar anaerobic capacity among players in different positions, which is consistent with the results of this hypothesis. However, we also see that in this study, no significant difference was observed between the anaerobic power of defenders and midfielders. ${ }^{9-11}$

The results show that there is no significant difference between the anaerobic power of defenders and attackers. According to the results, we find that the average anaerobic power of attackers is lower than other players and is significantly lower than the overall average obtained. We have already said that in foreign research, invaders have higher anaerobic power scores than some groups, which is not consistent with our results, and we have listed the reasons for this. Here we emphasize the fact that attackers have to face explosive power and ability to perform actions due to direct confrontation with defenders, and escaping from the defensive traps of the opposing team, using the spaces behind them and also succeeding in using air cannons. Develop speed significantly. However, in this study, the difference between the anaerobic capacity of attackers and defenders is not significant.

The results show that there is no significant difference between the anaerobic capacity of attackers and midfielders. The lack of significant differences in the anaerobic capacity of the two groups is a matter of course and is related to similar foreign research. The anaerobic capacity of young members of the Indian national team in different positions had differences, most of which were consistent with the present study. Despite these differences, the anaerobic capacity of attackers and midfielders in India has been significant, which does not correspond to the result of this assumption. The main reason that can be used to justify this distinction is the difference in the age range and championship level of Indian players compared to the players participating in this and other studies. As we said, the difference between the anaerobic power of attackers and midfielders in this study is not significant.

\section{CONCLUSION}

The position and the duration of activity has a great effect on $\mathrm{Vo}_{2 m a}{ }^{x}$ and anaerobic of players. So to increase both characteristics of the players depends on the position of the game. 


\section{REFERENCES}

1. Astorino, Todd. "Assessment of anaerobic power to verify VO2max attainment." Clinical Physiological Function Imaging. 30. (2010): 294-300. Print.

2. Dotan, Raffy. "Reverse Lactate Threshold:." International Journal of Sports Physiology and Performance. 7. (2012): 141-151. Print.

3. Faber, Nicholas. "Anaerobic Threshold Assessment Through the Ventilatory Method During Roller-Ski Skating Testing: Right or Wrong?." Journal of Strength and Conditioning Research. 26.2 (2012): 381-387. Print.

4. Fernandes, RJ."Step length and individual anaerobic threshold assessment in swimming..International Journal of Sports Medicine. 32.12 (2011): 940-946. Print.

5. Matthews, DA. "The anaerobic threshold: over-valued or under-utilized? A novel concept to enhance lipid optimization." Current Opinion In Clinical Nutrition And Metabolic Care. 15.5 (2012): 430-435. Print.

6. Jonathan M, Euan A. (1997) A perspective on exercise, lactate, and the anaerobic threshold. Chest 111:787-795. Joyner MJ, Coyle EF. (2008) Endurance exercise performance: The physiology of champions. J Physiol 586(1):35-44. Li FH, Li T, Ai JY,

7. Sun L, Min Z, Duan R, Zhu L, Liu YY, Liu TC. (2018) Beneficial autophagic activities, mitochondrial function, intensity interval training in a rat model, Front Physiol 9:571.

8. Mathews DM, Fox EL. The Physiological Basis of Physical Education and Athletics, Amazon. 1976.

9. McArdle WD, Katch FI, Katch VL. (1996) Exercise Physiology: Energy, Nutrition, and Human Performance. Baltimore, MD: Williams \&

10. Wilkins 316. Overend TJ, Paterson DH, Cunningham DA. (1992) The effects of interval and continuous training on the aerobic parameters. Can J Sport Sci 17(2):129-34.

11. Reilly T. (1997) Energetics of high-intensity exercise (soccer) with particular reference to fatigue. J Sports Sci 15:257-263.

12. Short KR, Vittone JL, Bigelow ML, Proctor DN, Nair KS. (2004) Age and aerobic exercise training effects on whole body and mucle protein metabolism. Am $\mathrm{J}$ Physiol Endocrinol Metab 286:E92-E101.

12. Ulupinar, S. (2020). Contribution of Energy Systems in Different Repetitive Sprint Protocols: Effect of Loading/Resting Ratios. Hacettepe University Institute of Health Sciences, Ankara

13. Temur H.B., (2018) Evaluation Of Muscle Damage And Changes In Some Blood Parameters Before And After An Intercolleguate Footbal Tournament, International Journal of Recent Scientific Research, Vol. 9, Issue, 4(B), pp. 2562825630 , 